\title{
Comparative Study between Extracts of Different Pomegranate Parts Issued from Five Tunisian Cultivars (Punica granatum L.): Phytochemical Content, Volatile Composition and Biological Activity
}

\author{
Manel Mekni ${ }^{*}$, Wafa Kharroubi ${ }^{1}$, Guido Flamimi ${ }^{2}$, Mariem Garrab ${ }^{3}$, \\ Maha Mastouri ${ }^{3}$ and Mohamed Hammami ${ }^{1}$
}

${ }^{1}$ Laboratory of 'Nutrition Functional Foods and Health Disease, Faculty of Medicine, University of Monastir, 5019, Tunisia

${ }^{2}$ Dipartimento di Chimica Bioogranica e Biofarmacia, Via Bonnano, 3365126 Pisa, Italy

${ }^{3}$ Laboratory of Microbiology, CHU Fattouma Bourguiba, 5000 Monastir, Tunisia

*Corresponding author

\begin{tabular}{|c|c|}
\hline & A B S T R A C T \\
\hline & \multirow{6}{*}{$\begin{array}{l}\text { Very few studies are interested to the volatile compounds of pomegranate and evoking } \\
\text { their potentials, especially, their antibacterial activity. In the present study, edible (arils) } \\
\text { and non-edible (peels) parts of five pomegranate varieties (Tounsi, Kalai, Zahri, Chelfi and } \\
\text { Garci) were compared for their phenolic contents, antioxidant activity, aroma profile and } \\
\text { antibacterial propriety of their volatile compounds. Results showed that peel of the five } \\
\text { varieties contained higher content in phenolics, flavonoids, tannins, anthocyanins and } \\
\text { carotenoids additionally to higher antioxidant power. Considerable correlations have been } \\
\text { highlighted between polyphenols, o-diphenols and flavonoids of peel extracts and the } \\
\text { DPPH scavenging activity as well as reducing power. Concerning volatile compounds, } \\
\text { Chelfi is quantitatively the richest cultivar. However, the Tounsi seems to be quantitatively } \\
\text { the richest for both peels and arils. Gram positive bacteria were more sensitive to volatiles } \\
\text { extract than gram negative with a substantial activity of both peel and seed extracts of } \\
\text { Tounsi variety. It's therefore tempting to speculate that the non-edible part (peel) has a } \\
\text { therapeutic and nutritional potential that must be valorised. }\end{array}$} \\
\hline Keywords & \\
\hline $\begin{array}{l}\text { Punica granatum, Peel, } \\
\text { Phytochemicals, } \\
\text { Antioxidant power, } \\
\text { Volatile compounds, } \\
\text { Antimicrobial activity }\end{array}$ & \\
\hline Artic & \\
\hline $\begin{array}{l}\text { Accepted } \\
12 \text { April } \\
\text { Availabl } \\
10 \text { May } 2\end{array}$ & \\
\hline & \\
\hline
\end{tabular}

\section{Introduction}

Pomegranate (Punica granatum L.), is one of the oldest cultivated fruit trees in Tunisia and in the worldwide. It is native from Iran to the Himalayas in northern India and has been cultivated and naturalized over the whole Mediterranean region since ancient times (Meerts et al., 2009). More than 500 pomegranate cultivars were identified worldwide, indeed high genetic diversity of morphological and biochemical quality traits characterize this plant (Da Silva et al., 2013).

Medicinal plants are rich source of traditional herbal medicine around the globe. Most of the plant's therapeutic properties are due to the presence of secondary bioactive compounds (Khan et al., 2017). For the pomegranate, several scientific studies have confirmed its 
biological activities and medicinal effects of its different parts (arils, peels, leaves, and flowers) and products (fresh and fermented juices, enriched extracts, and seed oil) (Lansky and Newman 2007; Aviram and Rosenbalt, 2012; Hassnaoui et al., 2014) given its high content of polyphenols (as tannins, ellagic acid, flavonoids, anthocyanins), fatty acids, sterols, terpenoids and alkaloids (Tehranifar et al., 2010; Mekni et al., 2013a). A tremendous attention was paid to pomegranate extracts as natural alternative for the treatment of a wide range of bacterial and viral infections due to their antimicrobial activity. Recent study indicates that both pomegranate aril and peel extracts have an effective antimicrobial activity, as evidenced by the inhibitory effect on the bacterial growth of two important human pathogens, including Staphylococcus aureus and Escherichia coli, often involved in food borne illness (Pagliarulo et al., 2016). Later the antimicrobial activity of extracts of pomegranate peel and juice on cariogenic bacteria has been also highlighted by Gianmaria Fabrizio Ferrazzano (Ferrazzano et al., 2017). Moreover MiklavčičVišnjevec et al., (2017) have reported the ethanol and water extracts of pomegranate exocarp and mesocarp showed the greatest antimicrobial activity against many bacterial strains tested.

In all these studies the presence of phytocompounds in the extracts including phenols, tannins and flavonoids as major active constituents that may be responsible for these activities. However, research studies dealing with volatiles components of pomegranate and evoking their antibacterial activity are almost absent, except a publication of Mekni et al., (2013b).

This prompted us to establish this study to investigate the aroma profile of peel and arils extracts of five Tunisian pomegranate varieties and to determine the antibacterial propriety of those volatile compounds, while quantifying phenolic (total phenolic Orthodiphenols, total flavonoid, total anthocyanins, condensed tannins)and non-phenolic compounds (carotenoids) and determining the antioxidant activity (DPPH,ATBSradical scavenging activity and reducing power).

\section{Materials and Methods}

\section{Preparation of pomegranate's methanol extracts}

Mature pomegranates from Tounsi, Kalai, Zahri, Chelfi and Garci cultivars were manually picked from different region of Tunisia. Samples were immediately peeled (arils were carefully separated by hand from the peel). Arils and peels were dried in oven at $40^{\circ} \mathrm{C}$ until constant weight, then were crushed and sieved to obtain fine powders and were extracted with methanol according to the method of Mau et al., (2001). All extracts were kept at $-20^{\circ} \mathrm{C}$ in the dark for further use.

\section{Phytochemical screening}

The methanolic extracts of peels and arils were tested for the presence of: total phenols, ortho-diphenols, total flavonoids, total anthocyanins, total condensed tannins and total carotenoids.

Total phenolic contents and O-diphenols of methanolic fractions were determined according to the method of Mekni et al., (2013a). Results were expressed on a dry weight (DW) basis as $\mathrm{mg}$ gallic acid equivalents (GAE) /g of sample.

Total flavonoid contents of the extracts were determined according to the colorimetric assay developed by Zhishen et al., (1999). The results were also expressed on a dry weight (DW) basis as mg catechin equivalents (CEQ)/ $\mathrm{g}$ of sample. 
Total anthocyanins were quantified according to the method of Giusti and Wrolstad (2000) with minor modifications. In brief, oneg of dried sample was extracted with $25 \mathrm{ml}$ of acidified methanol (1\% HCL) for $24 \mathrm{~h}$ at room temperature in the dark than centrifuged at $1000 x \mathrm{x}$ for $15 \mathrm{~min}$. Total anthocyanins content was determined as mg cyanidin 3glucoside equivalents (CyE) per $g$ of dry weight.

Determination of condensed tannins was based on the procedure reported by Sun et al., (1998) with slight modification. The amount of tannins was expressed as mg tannic acid /g of dry weight (mg TA/g DW) (Mekni et al., 2014).

Total carotenoids were extracted according to the method of Talcott and Howard (1999), with slight modifications (Mekni et al., 2013a).

Total carotenoids were calculated as follows and expressed as milligrams per $\mathrm{g}$ of dry weight $(\mathrm{DW})$. Total carotenoids $=\mathrm{Ab} \times \mathrm{V} \times$ $106 / \mathrm{A} 1 \% \times 100 \mathrm{G}$.

$\mathrm{Ab}$ is the absorbance at $470 \mathrm{~nm}, \mathrm{~V}$ is the total volume of extract, A $1 \%$ is the extinction coefficient for a $1 \%$ mixture of carotenoids at 2500 , and $\mathrm{G}$ is sample weight $(\mathrm{g})$.

\section{Antioxidant activity}

\section{DPPH radical scavenging activity}

Antioxidant activity of pomegranate extracts was evaluated through the measurement of the free-radical scavenging capacity by the DPPH. (2,2-diphenyl-1-picrylhydrazyl) assay (Kontogiorgis and Hadjipavlou-Litina, 2005).

The optical densities of the samples in the absence of DPPH were subtracted from the corresponding with DPPH. The reduction (\%) values were determined and compared to appropriate standards. Inhibition of the free radical DPPH is in per cent (I \%).

\section{ABTS free radical scavenging assay}

The ABTS assay was determined as described by Yvonne et al., (2005). The antioxidant capacity was calorimetrically determined where absorbance is measured at 734 nmandexpressed as \% of ABTS+ reduction.

\section{Reducing power}

The reducing powers of the studied extracts were determined according to the method described by Chung et al., (2005). The reducing powers of the tested samples increased with the absorbance values and expressed as $\mathrm{mg} / \mathrm{ml}$ of ascorbic acid (AA).

\section{Antibacterial activity}

To test the antimicrobial efficacy of the extracts, four bacterial strains were employed including Enterococcus faecalis ATCC 29212, Staphylococcus aureus ATCC 27950, Escherichia coli ATCC 25922 and Pseudomonas aeruginosa ATCC 27950 (microbial collections were chosen as important human pathogens). The antimicrobial activity was assessed through the determination of the minimal inhibitory concentration (MIC) by the microdilutionplate dilution method (Samania et al., 2016).

Each extract was dissolved in 10\% dimethyl sulfoxide (DMSO). The plant extract concentrations tested ranged from 1 to 10 $\mathrm{mg} / \mathrm{ml}$, added to the microbial culture.

After incubation at $37 \circ \mathrm{C}$ between 18 and 24 $\mathrm{h}$, the MIC of each extract was defined as the lowest concentration which inhibited bacterial growth, Results were expressed in milligram per millilitre. 


\section{Extraction of volatiles and GC-MS analysis}

According to the method of Mekni et al., (2013b), $100 \mathrm{~g}$ of the fine powders of pomegranate arils and peels of each sample was subjected to hydro distillation for $4 \mathrm{~h}$ using a Clevenger-type apparatus (Clevenger, 1928).

The volatiles obtained after trapping in hexane were dried over anhydrous sodium sulphate, evaporated and concentrated under a gentle stream of $\mathrm{N}_{2}$ and stored at $-20{ }^{\circ} \mathrm{C}$ until further analysis. GC analyses were accomplished according to the method described by Mekni et al., (2013b).

\section{Statistical analyses}

All data were subjected to analyses of variance (ANOVA one-way) using statistical package for social sciences (SPSS) 11.0 software (SPSS, Chicago, IL, USA). The data shown are mean values $(n=3)$ and the significance of the differences was compared using Duncan multiple range test at $p<0.05$ probability level. Simple associations between variables were calculated as the Pearson correlation. Principal component analysis (PCA) was carried out and all parameters analysed were used as variables in PCA using XLStat-Pro version 7.5.2007 for Windows (Addinsoft, New York, USA).

\section{Results and Discussion}

\section{Phenolic and non-phenolic compounds content}

Results concerning the differences between the amounts of phenolic compounds (including total polyphenols, O-diphenols, flavonoids, anthocyanins and tannins) and non-phenolics compounds (carotenoids) in arils and peels extracts, of the five pomegranate varieties are registered in Table 1.
The contents are variable according to the cultivar and the tested organ.

The highest amounts of total polyphenolic compounds (TPC) were recorded in peel extracts in all cultivars. Indeed, the peels extracts contain average twenty-eight-fold higher TPC than arils extracts for the five tested varieties. Similar findings were observed by Amri et al., (2017), suggesting that differences in TPC between peels and arils can be due to genetic variability which is involved in modification of the biosynthesis path of secondary phenolic metabolites. Furthermore, the variety Chelfi showed the highest amount of TPC followed by the varieties Garsi, Zahri, Kalai and Tounsi. These observations, underline the therapeutic and nutritional importance of pomegranate peels. Our results corroborate with numerous studies suggesting that pomegranate peel extract has more potential for health as a natural antioxidant-rich dietary supplement than arils extract. In previous studies Gill et al., (2000) than Tzulker et al., (2007) deduced that the antioxidant activity of pomegranate juice with higher levels of peel residues was two to twenty times higher than pomegranate juice prepared only with arils.

Similarly, for total orthodi-phenols (TOP), the levels vary significantly from one variety to another, and the levels found in peels are eighteen times higher than in arils (Table 1). The highest TOP concentration was recorded in peel extract for Kalai variety followed by Zahri than Chelfiand Garci while the lowest TOP level was found in Tounsi variety. Comparing the O-diphenol levels in the arils, the Chelfi variety contains the higher concentration with $0.57 \mathrm{mg} / \mathrm{g}$ DM ( $\mathrm{p}<0.05$ ) followed by Garsi and Kalai varieties $(0.48$ and $0.42 \mathrm{mg} / \mathrm{g}$ MS, respectively). Both varieties Tounsi and Zahri present, statistically, the lowest amounts. The levels of O-diphenols vary according to the tested cultivar and organ. These results agree with 
those reported by Zaouay et al., (2012) and those of Amri et al., (2017) suggesting that the TOP contained in the peel is more important than in juice of both Tounsi and Nana varieties.

Total flavonoids content (TFC) is summarized in Table 1. Significant differences in flavonoids levels were found between organs and varieties. TFC in peels are up to ten times stronger than those in arils. Maximum levels of Totals flavonoids are obtained in peels of the Garsi variety (15.13 mg / g DM, p <0.05) followed by Zahri, Chelfi, Kalai and Tounsi varieties in descending order $(p<0.05)$. The differences in TFC between cultivars may be related to fruit type (sweet and sour) and geographical area of origin (li et al., 2015) also it can be associated with the difference in the stage of maturity of cultivars (Gullon et al., 2016; Pagliarulo et al., 2016). Indeed, total polyphenol levels increase at the early stage of growth in peel, but thereafter generally decrease during maturation (Saad et al., 2012). Regarding arils, low levels of flavonoids are recorded in all varieties studied without any significant difference noted and these contents vary from $1.32 \mathrm{mg} / \mathrm{g}$ at Chelfi variety up to $2.59 \mathrm{mg} / \mathrm{g} \mathrm{DM}$ at Garsi variety. Our results agree with those of $\mathrm{Li}$ et al., (2006) who reported that pomegranate peels extracts contain much higher levels of flavonoids than those found in arils extracts.

Considerable levels of tannins in both arils and peel of pomegranate are detected, suggesting that this compound contribute to the antioxidant power of these fruits. This power is attributed probably to the effect of hydrolysable tannins and an ellagitannin, known as punicalagin, present in all pomegranate cultivars (Tzulker et al., 2007). That's why significant inter-cultivar and interorgan variation was revealed in Table 1. Indeed, the arils of Tounsi variety and the peels of the Zahri variety are noted the highest in condensed tannins (7.85 and $7.47 \mathrm{mg} / \mathrm{g}$ DM, p <0.05, respectively), followed by peels of Garsi and Tounsi varieties (6.96 and 6.19 mg / g DM, respectively, p <0.05). On the other hand, the arils and peels of Chelfi and Kalai varieties provided the lowest levels (between 3.73 and $5.11 \mathrm{mg} / \mathrm{g} \mathrm{DM}, \mathrm{p}<0.05$ ).

The appealing colour of pomegranate juice, an important index for juice quality and popularity, is mainly attributed to anthocyanin concentration; they are almost exclusively responsible forred, blue and purple colours in fruits (Zhang et al., 2011). And healthy functions of anthocyanins have been widely recognized and studied (Stinzting and Carle 2004). In the present work the total anthocyanin concentration of the five pomegranate cultivars varied from 0.15 to $6.88 \mathrm{mg} / \mathrm{g}$ DM. The total anthocyanin concentration was found to be more abundant in peels, three times higher, than in arils variety (Table 1). Peels of Tounsi variety are the richest in anthocyanins followed by those of the Zahri variety. On the other hand, Chelfi variety showed the lowest amount among studied cultivars. The arils of the Chelfi variety are the richest in anthocyanins followed by Tounsi and Kalai varieties. However, the lowest level is recorded inZahri variety. These results are consistent with the data reported by Tomas-Barberan et al., (2001), who found that fruit peels such as peaches and Plums usually contain larger quantities of phenolic compounds, anthocyanins and flavonoids. Total anthocyanins sequential order of five tested pomegranate varieties, was consistent with that of all phenolic compounds, their levels were affected by similar factors, including growth environment, cultivar, maturity and seasonal conditions (li et al., 2015, Gil et al., 1995, Borohov-Neori et al., 2009).

Referring to Table 1, there are statistically significant differences between the total 
carotenoid levels in all the varieties concerned, whether in arils or peels. In fact, the highest levels are reported in arils of Tounsi variety followed by peels of Garsi and Zahri varieties $(\mathrm{p}<0.05)$. Peels of Tounsi and Kalai varieties come in third place with no statistical difference. Lastly, come the arils of Zahri and Chelfi varieties.

Previous results suggest that Tounsi, Zahri, and Gabsi varieties primarily generate pigmentation compounds such as carotenoids, anthocyanins and tannins. While the Kalai and Chelfi varieties mainly provide polyphenols, O-diphenols and flavonoids.

For better visualization, a principal component analysis (PCA) based on the distribution of phenolic compounds and carotenoids according to the organs (arils and peels) of five different Tunisian varieties of pomegranate Punica granatum $\mathrm{L}$. is carried out (Fig. 1a). According to the PCA, the total variance explained by the first two axes is $79.78 \%$.

The principal axis $\mathrm{PC} 1$, is positively correlated, in one hand, with phenolic compounds as well as carotenoids and on the other hand, with the peels of all studied varieties. In parallel, two groups are also separated along the same axis suggesting that Tounsi, Zahri, and Gabsi varieties primarily provide pigmentation compounds such as carotenoids, anthocyanins and tannins. While the Kalai and Chelfi varieties mainly yield polyphenols, O-diphenols and flavonoids. However, the arils of the different studied varieties are negatively associated to PC1. Based on previous results, the most part of the nutritional value and therapeutic contribution of pomegranate fruit seem to be mostly provided by the inedible part "peels", while the edible part "arils" would guarantee a low content of phenolic compounds compared to peels.

\section{Antioxidant activity}

Owing to its richness in polyphenols, pomegranate peels are known to possess strong antioxidant activity. DPPH, ATBS free radicals scavenging and reducing power, three colorimetric methods were used to evaluate the antioxidant activity of different extracts.

In accordance with the high content in polyphenols, pomegranate peel extracts showed very high antioxidant activity in all tests (Figure 2).

For DPPH assay, as described in the Figure $2 \mathrm{a}$, the most important antiradical capacity is noted in the peel extracts of Garsi, Chelfi, Zahri and Kalai varieties with no statistically significant difference, followed by peel extract of Tounsi variety $(\mathrm{p}<0.05)$.

For all tested samples, arils extracts show lower antioxidant potency than peels, except for Garsi variety having similar inhibition percentage to that recorded in peels. Our results agree with those reported by Tehranifar et al., (2011) and Li et al., (2006).

As described by the results of the ABTS assay (Fig. 2b), an effective and potent antioxidant power for both organs (peels and arils) in the tested varieties is registered. These results clearly show the antioxidant effect of Tunisian pomegranate arils and peels.

This effect is previously reported by Zaouay et al., (2012) who tested the antioxidant potency of Tunisian pomegranate varieties by the ABTS method, they suggest that pomegranate juice extracts have a very considerable antioxidant potential.

The reducing powers of the different studied pomegranate varieties are illustrated in Figure 2c. Indeed, peels extracts provided the strongest antioxidants for all tested varieties. 
Table.1 Phytochemicals (mg/g GAE, DW) contents in extracts of different organs in five pomegranate varieties. Values are expressed as means \pm standard deviation $(n=3)$. Means with different letters were significantly different at the level of $\mathrm{p}<0.05$.

\begin{tabular}{|c|c|c|c|c|c|c|c|}
\hline Varieties & Organs & Polyphenols & Anthocyanins & Tannins & Orthodiphenols & Carotenoids & Flavonoids \\
\hline \multirow[t]{2}{*}{ Tounsi } & Arils & $3.33 \pm 0.55^{\mathrm{a}}$ & $2.6 \pm 0.36^{\mathrm{c} . \mathrm{d}}$ & $7.85 \pm 0.45^{\mathrm{f}}$ & $0.35 \pm 0.04^{\mathrm{a}}$ & $3.85 \pm 0.4^{\mathrm{e}}$ & $2.89 \pm 0.21^{\mathrm{a}}$ \\
\hline & peels & $104.79 \pm 5.5^{\mathrm{b}}$ & $6.88 \pm 0.68^{f}$ & $6.19 \pm 0.96^{\text {d.e }}$ & $2.4 \pm 0.08^{c}$ & $2.3 \pm 0.37^{\mathrm{c} . \mathrm{d}}$ & $12.11 \pm 1.17^{\mathrm{b}}$ \\
\hline \multirow[t]{2}{*}{ Kalai } & Arils & $5.86 \pm 0.29^{\mathrm{a}}$ & $2.62 \pm 0.11^{\text {c.d }}$ & $4.56 \pm 0.17^{a . b}$ & $0.42 \pm 0.08^{\mathrm{a} . \mathrm{b}}$ & $2.26 \pm 0.32^{\text {c.d }}$ & $1.9 \pm 0.22^{\mathrm{a}}$ \\
\hline & peels & $114.44 \pm 11.46^{\mathrm{c}}$ & $3.25 \pm 0.29^{\mathrm{d}}$ & $3.73 \pm 0.34^{\mathrm{a}}$ & $2.67 \pm 0.03^{\mathrm{d}}$ & $2.34 \pm 0.27^{\text {c.d }}$ & $12.74 \pm 0.58^{\text {b.c }}$ \\
\hline \multirow[t]{2}{*}{ Zahri } & Arils & $4.56 \pm 0.61^{\mathrm{a}}$ & $1.67 \pm 0.07^{b}$ & $5.85 \pm 0.85^{\mathrm{c.d}}$ & $0.36 \pm 0.02^{\mathrm{a}}$ & $0.84 \pm 0.18^{\mathrm{a}}$ & $1.58 \pm 0.07^{\mathrm{a}}$ \\
\hline & peels & $133.39 \pm 18.04^{c}$ & $4.38 \pm 0.87^{\mathrm{e}}$ & $7.47 \pm 0.06^{\mathrm{t}}$ & $2.66 \pm 0.09^{d}$ & $2.61 \pm 0.06^{\mathrm{d}}$ & $14.06 \pm 0.85^{\mathrm{cdd}}$ \\
\hline \multirow[t]{2}{*}{ Chelfi } & Arils & $5.42 \pm 1.01^{\mathrm{a}}$ & $3.29 \pm 0.58^{\mathrm{d}}$ & $5.11 \pm 0.42^{b . c}$ & $0.57 \pm 0.25^{\mathrm{b}}$ & $0.77 \pm 0.11^{\mathrm{a}}$ & $1.32 \pm 0.01^{\mathrm{a}}$ \\
\hline & peels & $143.98 \pm 9.84^{\mathrm{c}}$ & $0.15 \pm 0.01^{\mathrm{a}}$ & $4.22 \pm 0.15^{a . b}$ & $2.6 \pm 0.03^{d}$ & $1.94 \pm 0.14^{\mathrm{c}}$ & $13.6 \pm 0.86^{\text {b.c.d }}$ \\
\hline \multirow[t]{2}{*}{ Garci } & Arils & $4.49 \pm 0.47^{\mathrm{a}}$ & $2.06 \pm 0.11^{\text {b.c }}$ & $4.42 \pm 0.87^{\text {a.b }}$ & $0.48 \pm 0.04^{\text {a.b }}$ & $1.39 \pm 0.19^{b}$ & $2.59 \pm 0.25^{\mathrm{a}}$ \\
\hline & peels & $134.44 \pm 7.19^{c}$ & $2.7 \pm 0.29^{\text {c.d }}$ & $6.96 \pm 0.42^{\text {e.f }}$ & $2.6 \pm 0.08^{\mathrm{d}}$ & $2.63 \pm 0.48^{\mathrm{d}}$ & $15.13 \pm 1.96^{\mathrm{d}}$ \\
\hline
\end{tabular}

Table. 2 Correlations between bioactive compounds and the antioxidant power of arils and peels of five Tunisian pomegranate varieties. ** very significant correlation $<0.01$

\begin{tabular}{|c|c|c|c|}
\hline $\begin{array}{c}\text { Correlation of } \\
\text { Pearson }\end{array}$ & DPPH & ABTS & Reducing Power \\
\hline Polyphenols & $\mathbf{0 . 4 9 7 * *}$ & 0.181 & $\mathbf{0 . 6 4 5}^{* *}$ \\
\hline O-diphenols & $\mathbf{0 . 6 4 1} * *$ & 0.195 & $\mathbf{0 . 8 7 8}^{* *}$ \\
\hline Flavonoids & $\mathbf{0 . 5 7 1} * *$ & 0.24 & $\mathbf{0 . 8 9 3}^{* *}$ \\
\hline Anthocyanins & 0.128 & -0.045 & -0.036 \\
\hline Tannins & -0.147 & -0.094 & 0.102 \\
\hline Carotenoids & -0.292 & 0.115 & 0.126 \\
\hline
\end{tabular}

Table.3 Volatile compounds (\%) evaluated by GC-MS in arils and peel extracts of five Punica granatum $\mathrm{L}$. varieties

\begin{tabular}{|l|}
\multicolumn{1}{|c|}{$\begin{array}{c}\text { Constituent } \\
(\%)\end{array}$} \\
\hline Alcohols \\
\hline Isopentyl alcohol \\
2,3-butanediol \\
\hline 1,3-butanediol \\
\hline (E) -3-hexen-1-ol \\
\hline 1-hexanol \\
\hline 1-heptanol \\
\hline 1-octanol \\
\hline $\begin{array}{l}\text { Cis-linalool oxide } \\
\text { (furanoid) }\end{array}$ \\
\hline
\end{tabular}

\begin{tabular}{|c|c|c|c|c|c|c|c|c|c|c|}
\hline & l.r.i & \multicolumn{2}{|c|}{ Tounsi } & \multicolumn{2}{|c|}{ Kalai } & \multicolumn{2}{|c|}{ Garsi } & \multicolumn{2}{|c|}{ Zahri } & \multicolumn{2}{|c|}{ Chelfi } \\
\hline & Peels & Arils & Peels & Arils & Peels & Arils & Peels & Arils & Peels & Arils \\
\hline $\mathbf{7 4 1}$ & - & - & - & - & - & - & - & - & - & - \\
\hline $\mathbf{8 0 4}$ & - & - & - & - & - & - & - & - & - & - \\
\hline $\mathbf{8 1 5}$ & - & - & tr & 2 & - & 1.3 & 3.8 & 17.3 & 6.2 & 31.5 \\
\hline $\mathbf{8 5 2}$ & - & - & 0.7 & - & - & - & - & - & - & - \\
\hline $\mathbf{8 6 8}$ & - & - & - & - & - & - & - & - & - & - \\
\hline $\mathbf{9 7 0}$ & - & tr & - & - & - & - & - & - & - & - \\
\hline $\mathbf{1 0 7 1}$ & 0.2 & - & - & - & - & - & - & - & - & - \\
\hline $\mathbf{1 0 7 5}$ & - & - & - & - & - & - & 1.3 & - & 1.6 & - \\
\hline
\end{tabular}




\begin{tabular}{|c|c|c|c|c|c|c|c|c|c|c|c|}
\hline Phenylethyl alcohol & 1111 & - & - & - & 1 & - & - & 2.3 & 5.3 & 0.9 & - \\
\hline Trans-carveol & 1220 & - & - & - & - & - & - & - & 2.4 & - & - \\
\hline 1-decanol & 1273 & - & - & - & - & - & - & - & - & 1.3 & - \\
\hline Dodecanol & 1471 & - & - & - & 0.3 & - & - & - & - & - & - \\
\hline$\%$ identified & & $0.2 \%$ & $1.9 \%$ & $0.7 \%$ & $3.6 \%$ & - & - & $7.4 \%$ & $25 \%$ & - & - \\
\hline Esters & & & & & & & & & & & \\
\hline Ethyl acetate & 618 & - & - & - & 1.6 & - & & - & - & - & - \\
\hline Allyl butyrate & 884 & - & - & - & - & - & 0.6 & - & - & - & - \\
\hline Methyl pentanoate & 828 & - & 1 & - & - & - & 1 & - & 14.1 & - & - \\
\hline Ethyl pentanoate & 902 & - & 0.6 & - & - & - & 0.4 & - & - & 0.4 & - \\
\hline $\mathrm{N}$-pentyl acetate & 912 & - & 0.4 & - & - & - & 2.3 & - & - & & - \\
\hline Methyl hexanoate & 930 & 3.5 & 0.5 & 1.2 & - & 1 & 0.7 & - & - & 0.7 & - \\
\hline Ethyl hexanoate & 998 & - & 0.3 & - & - & - & - & - & - & & - \\
\hline 1-hexyl acetate & 1009 & - & - & - & - & - & 0.1 & - & - & 0.1 & - \\
\hline Butyl pentanoate & 1094 & - & 1.4 & - & - & - & - & - & \multicolumn{2}{|c|}{0.5} & - \\
\hline $\begin{array}{l}\text { 3-methylbutyl 2- } \\
\text { methylbutanoate }\end{array}$ & 1101 & - & 0.2 & - & - & - & 0.2 & - & - & 0.2 & - \\
\hline 3-octyl acetate & 1125 & - & - & - & - & - & 0.3 & - & - & 0.3 & - \\
\hline Methyl octanoate & 1129 & 2.9 & 0.9 & 16.9 & 27.1 & 14.8 & 20 & 10.1 & 0.9 & 5.8 & 3.2 \\
\hline Isopentyl n-butyrate & 1060 & & 0.5 & - & - & - & - & - & - & - & - \\
\hline Ethyl benzoate & 1171 & 0.2 & - & - & & - & - & - & & & - \\
\hline $\begin{array}{l}\text { Methyl ester of } \\
\text { benzenic acetic acid }\end{array}$ & 1179 & - & - & - & - & - & 4 & - & - & 4 & - \\
\hline n-butyl n-hexanoate & 1189 & - & 6.6 & - & - & - & - & - & - & - & - \\
\hline Ethyl octanoate & 1195 & 1.1 & 1 & - & 3.6 & - & 6.9 & - & - & 6.9 & - \\
\hline $\begin{array}{l}\text { 2-phenyl ethyl } \\
\text { acetate }\end{array}$ & 1258 & - & - & - & 0.8 & - & 2.5 & - & 1.5 & 2.5 & 1 \\
\hline $\begin{array}{l}\text { Trans-dihydro-a- } \\
\text { terpinyl acetate }\end{array}$ & 1301 & 1.1 & - & - & - & - & - & - & - & - & - \\
\hline $\begin{array}{l}\text { Dihydrocitronellol } \\
\text { acetate }\end{array}$ & 1321 & 0.3 & - & - & - & - & - & - & - & 0.4 & - \\
\hline Methyl decanoate & 1328 & 1.7 & 0.5 & 6.8 & 13 & 12.4 & 11.1 & 11.6 & 1 & 4.1 & - \\
\hline Methyl nonanoate & 1227 & 0.6 & - & 1.4 & 0.9 & 1.6 & 0.7 & 1.2 & - & 0.8 & - \\
\hline Ethyl nonanoate & 1294 & 0.3 & - & - & & & 0.1 & & & 0.1 & \\
\hline Methyl dodecanoate & 1526 & - & - & - & 0.8 & 4.3 & 1.2 & 10.5 & 2.8 & 1.2 & 1.3 \\
\hline Pentyl octanoate & 1490 & - & 0.3 & - & - & - & - & - & - & - & - \\
\hline $\begin{array}{l}\text { Methyl } \\
\text { tetradecanoate }\end{array}$ & 1726 & - & 0.2 & - & - & 1.9 & 0.4 & 2.2 & 2.2 & 0.4 & 2.2 \\
\hline $\begin{array}{l}\text { Ethyl } \\
\text { tetradecanoate }\end{array}$ & 1793 & - & - & 1.2 & - & - & - & - & 0.2 & - & 0.2 \\
\hline $\begin{array}{l}\text { Methyl } \\
\text { hexadecanoate }\end{array}$ & 1927 & - & 0.2 & 7.2 & 0.2 & 24.9 & 1 & 17.8 & - & 1 & - \\
\hline
\end{tabular}




\begin{tabular}{|c|c|c|c|c|c|c|c|c|c|c|c|}
\hline $\begin{array}{l}\text { Methyl 9- } \\
\text { octadecenoate }\end{array}$ & 2116 & - & - & 4.7 & - & - & - & - & - & - & - \\
\hline Ethyl decanoate & 1395 & - & - & - & 0.8 & - & 1.3 & - & 0.3 & - & 0.3 \\
\hline$\%$ identified & & $11.7 \%$ & $15.6 \%$ & $39.4 \%$ & $48.8 \%$ & $60.9 \%$ & $54.8 \%$ & $53.4 \%$ & $23.5 \%$ & $30.5 \%$ & $8.2 \%$ \\
\hline \multicolumn{12}{|l|}{ Aldehydes } \\
\hline Hexanal & 802 & 1.6 & 2.1 & - & - & - & - & - & - & - & - \\
\hline Furfural & 831 & 2.9 & $\operatorname{tr}$ & 2.1 & 17 & - & 20.6 & - & - & 4 & 15.8 \\
\hline (E) -2-hexenal & 855 & - & 0.9 & - & - & - & - & - & - & - & - \\
\hline Benzaldehyde & 962 & - & & $\operatorname{tr}$ & - & - & - & - & - & - & - \\
\hline (Z) -2-heptenal & 963 & - & 4.3 & - & - & - & - & - & - & - & - \\
\hline 5-methyl furfural & 964 & 0.4 & & - & 0.7 & - & 0.6 & - & 1 & 0.7 & 0.9 \\
\hline Octanal & 1002 & 0.7 & 0.5 & - & - & - & - & - & - & - & - \\
\hline $\begin{array}{l}\text { (E.e.) - } \\
\text { 2.4heptadienal }\end{array}$ & 1015 & 0.4 & - & - & - & - & - & - & - & - & - \\
\hline (E) -2-octenal & 1063 & - & 1.1 & - & - & - & - & - & - & - & - \\
\hline Nonanal & 1103 & 2.6 & 1.6 & 1.8 & .07 & 1.7 & .05 & 1.8 & 1.9 & 3.4 & 3.8 \\
\hline $\begin{array}{l}(\mathrm{E}, \mathrm{E}) \text {-2,4- } \\
\text { octadienal }\end{array}$ & 1110 & - & $\operatorname{tr}$ & - & - & - & - & - & - & - & - \\
\hline Decanal & 1205 & 2.2 & 0.2 & 2.4 & 0.3 & 1.7 & 0.3 & 2.2 & 5.9 & 0.6 & - \\
\hline $\begin{array}{l}\text { 2-ethyl- } \\
\text { benzaldehyde }\end{array}$ & 1208 & 5.8 & - & 2.7 & - & - & - & - & - & 7 & - \\
\hline $\begin{array}{l}(\mathrm{E}, \mathrm{E})-\mathbf{2 , 4 -} \\
\text { nonadienal }\end{array}$ & 1218 & - & 11.8 & - & - & - & 6.1 & - & 8 & 6.1 & 8 \\
\hline (Z) -4-DECENAL & 1194 & - & 16.3 & - & - & - & - & - & - & - & - \\
\hline (E) -2-decenal & 1261 & - & 0.4 & - & - & - & - & - & - & - & - \\
\hline Undecanal & 1306 & 0.6 & 0.2 & - & - & - & - & - & 1.1 & 7.1 & 3.5 \\
\hline 2-undecanal & 1368 & - & 0.7 & - & - & - & - & - & - & - & - \\
\hline Dodecanal & 1407 & - & - & - & $\operatorname{tr}$ & - & - & - & 1 & 0.8 & 1 \\
\hline Tetradecanal & 1611 & - & - & - & $\operatorname{tr}$ & - & - & - & - & - & - \\
\hline$\%$ identified & & $17.8 \%$ & $40.1 \%$ & $9 \%$ & $18.4 \%$ & $1.7 \%$ & $28.1 \%$ & $1.8 \%$ & $18.9 \%$ & $21.8 \%$ & $33 \%$ \\
\hline \multicolumn{12}{|l|}{ Terpenoids } \\
\hline a-pinene (alpha) & 932 & $\operatorname{tr}$ & - & - & - & - & - & - & - & - & - \\
\hline a-terpinene (alpha) & 1018 & $\operatorname{tr}$ & - & 0.5 & - & - & - & - & - & $\operatorname{tr}$ & - \\
\hline o-cymene (ortho) & 1023 & - & - & 1 & - & - & - & - & - & - & - \\
\hline p-cymene (para) & 1026 & 0.3 & 0.2 & & 0.3 & - & - & - & - & $\operatorname{tr}$ & - \\
\hline Limonene & 1031 & - & 0.6 & 2.9 & 2.9 & - & 0.6 & 1.4 & 1.3 & - & - \\
\hline 1,8-cineole & 1033 & 4 & 0.8 & 2.9 & & - & - & 2.2 & 0.2 & - & - \\
\hline $\begin{array}{l}\text { g-terpinene } \\
\text { (gamma) }\end{array}$ & 1062 & 1.8 & - & 1 & 2 & - & - & - & - & - & - \\
\hline $\begin{array}{l}\text { p-mentha-2,4 (8) - } \\
\text { diene }\end{array}$ & 1086 & - & - & - & - & - & - & - & - & 3.1 & - \\
\hline Terpinolene & 1089 & 0.8 & - & 1.8 & 0.7 & - & 0.2 & - & - & - & - \\
\hline
\end{tabular}




\begin{tabular}{|c|c|c|c|c|c|c|c|c|c|c|c|}
\hline Linalool & 1098 & 1.6 & - & 0.7 & & 0.6 & & 1.4 & - & 1.7 & 0.5 \\
\hline $\begin{array}{l}\text { 1,3,8-p- } \\
\text { menthatriène }\end{array}$ & 1112 & $\operatorname{tr}$ & - & - & - & - & - & - & - & - & - \\
\hline Camphor & 1144 & - & - & 0.7 & - & 0.5 & - & 0.8 & - & 0.8 & - \\
\hline Menthol & 1174 & - & - & - & - & & - & 0.7 & - & - & 0.2 \\
\hline g-terpineol (gamma) & 1178 & 14.5 & 1.1 & 6.8 & - & 5.1 & - & 8.6 & 5.1 & 14.1 & 8.7 \\
\hline p-cymen-8-ol (para) & 1184 & 0.5 & - & - & - & & - & & 0.2 & $\operatorname{tr}$ & 0.2 \\
\hline a-terpineol (alpha) & 1190 & 1.3 & - & 0.6 & - & 0.9 & - & 1.4 & 0.3 & 1.9 & 0.5 \\
\hline Dihydro citronellol & 1196 & 1 & - & - & - & - & - & - & - & 3.6 & - \\
\hline Verbenone & 1207 & 0.4 & - & - & - & - & - & - & - & $\operatorname{tr}$ & - \\
\hline Pulegone & 1237 & - & 0.9 & - & - & - & - & - & - & $\operatorname{tr}$ & - \\
\hline (E) -anéthole & 1284 & 1.4 & - & 0.9 & - & - & $\operatorname{tr}$ & 1.3 & 0.4 & 2.4 & 0.4 \\
\hline cis- $\alpha$-bergamotene & 1416 & 0.4 & - & - & - & - & - & - & - & - & - \\
\hline $\begin{array}{l}\beta \text {-caryophyllene } \\
\text { (beta) }\end{array}$ & 1418 & 2.3 & 0.1 & 1.4 & $\operatorname{tr}$ & 1.3 & 0.5 & - & 1.4 & 4.5 & 2.9 \\
\hline $\begin{array}{l}\text { Trans- } \alpha- \\
\text { bergamotene }\end{array}$ & 1439 & 1.8 & 0.3 & 1 & 0.6 & 0.8 & 0.1 & 1.5 & 1.2 & 2.4 & 0.3 \\
\hline (E) -géranylacétone & 1454 & 4 & - & 2.3 & 0.2 & 1.7 & - & 1 & 2.7 & 2.6 & 0.5 \\
\hline$\beta$-selinene (beta) & 1485 & 1 & - & - & - & - & - & - & - & - & - \\
\hline$\%$ identified & & $39.7 \%$ & $4 \%$ & $24.5 \%$ & $6.7 \%$ & $10.9 \%$ & $1.4 \%$ & $20.3 \%$ & $12.8 \%$ & $37.1 \%$ & $15.5 \%$ \\
\hline \multicolumn{12}{|l|}{ Ketones } \\
\hline $\begin{array}{l}\text { 6-methyl-5-Hepten- } \\
\text { 2-one }\end{array}$ & 986 & 1 & - & 0.9 & - & 1.1 & - & - & - & 0.7 & - \\
\hline 3-octen-2-one & 1040 & - & 0.3 & - & - & - & - & - & & - & - \\
\hline 2-acetyl pyrrole & 1061 & - & & - & - & - & - & - & 0.4 & 0.2 & - \\
\hline 5-nonan & 1074 & - & 1 & - & - & - & - & - & - & - & - \\
\hline Fenchone & 1088 & - & & - & - & 2.5 & - & - & - & - & - \\
\hline 3-nonanone & 1140 & - & 1.4 & - & - & - & - & - & - & - & - \\
\hline 5-decanone & 1162 & - & 0.7 & - & - & - & - & - & - & - & - \\
\hline 2-undecanone & 1292 & - & & - & - & - & - & - & - & - & - \\
\hline Acetophenone & 1066 & 0.3 & - & - & - & - & - & - & - & $\operatorname{tr}$ & - \\
\hline$\%$ identified & & $1.3 \%$ & $3.4 \%$ & $0.9 \%$ & - & $3.6 \%$ & - & - & $0.4 \%$ & $0.9 \%$ & - \\
\hline Lactones & & - & - & - & - & - & - & - & - & - & - \\
\hline g-nonalactone & & - & - & - & - & - & - & - & $0.4 \%$ & - & - \\
\hline$\%$ identified & & - & - & - & - & - & - & - & $0.4 \%$ & - & - \\
\hline \multicolumn{12}{|l|}{$\begin{array}{l}\text { Saturated } \\
\text { Hydrocarbon }\end{array}$} \\
\hline n-undecane & 1099 & - & - & - & - & - & 0.3 & - & - & - & - \\
\hline n-dodecane & 1199 & 1 & - & - & - & - & - & - & - & - & - \\
\hline n-tridecane & 1299 & 1.3 & 0.2 & 1 & 0.2 & & 0.7 & 0.7 & 0.5 & 1.3 & - \\
\hline n-tetradecane & 1399 & 13.4 & 5.2 & 5.9 & 2.5 & 3.3 & 3.6 & 3.3 & 3.9 & 10.7 & - \\
\hline n-pentadecane & 1500 & 1.1 & - & - & - & - & - & - & - & 1.3 & - \\
\hline n-hexadecane & 1600 & 1.8 & - & 1.5 & - & 1.9 & 0.6 & - & - & - & - \\
\hline
\end{tabular}




\begin{tabular}{|c|c|c|c|c|c|c|c|c|c|c|c|}
\hline n-heptadecane & 1700 & 1 & 0.2 & - & - & 0.8 & - & - & - & - & - \\
\hline$\%$ identified & & $19.6 \%$ & $5.6 \%$ & $8.4 \%$ & $2.7 \%$ & $6 \%$ & $5.2 \%$ & $4 \%$ & $4.4 \%$ & $13.3 \%$ & - \\
\hline \multicolumn{12}{|l|}{$\begin{array}{l}\text { Unsaturated } \\
\text { hydrocarbons }\end{array}$} \\
\hline 1-decene & 991 & 0.3 & 1 & - & - & 1.5 & - & - & - & - & - \\
\hline 1-tetradecene & 1392 & - & 0.3 & - & - & - & - & - & - & - & - \\
\hline$\%$ identified & & $0.3 \%$ & $1.3 \%$ & - & - & $1.5 \%$ & - & - & - & - & - \\
\hline \multicolumn{12}{|l|}{ Carboxylic acids } \\
\hline Pentanoic acid & 900 & - & 17.1 & - & - & - & - & - & 1.5 & - & 1.9 \\
\hline Hexanoic acid & 985 & 1.8 & 3.2 & - & - & - & - & - & - & - & - \\
\hline Octanoic acid & 1173 & - & - & 1 & 10.3 & - & - & - & - & - & - \\
\hline Nonanoic acid & 1280 & - & - & - & 0.2 & - & - & - & - & - & - \\
\hline Decanoic acid & 1379 & - & - & - & 0.7 & - & - & - & 0.3 & 0.2 & - \\
\hline$\%$ identified & & $1.8 \%$ & $20.3 \%$ & $1 \%$ & $11.2 \%$ & $0 \%$ & $0 \%$ & $0 \%$ & $1.8 \%$ & $0.2 \%$ & $1.9 \%$ \\
\hline \multicolumn{12}{|l|}{ Other classes } \\
\hline 2-butyl furan & 892 & - & - & - & - & - & 0.3 & - & - & - & - \\
\hline 2-acetyl furan & 905 & - & 1.4 & 1.4 & & 0.7 & - & - & 2.6 & 0.8 & 2.9 \\
\hline $\begin{array}{l}\text { 2,5-dimethyl } \\
\text { pyrazine }\end{array}$ & 911 & - & - & - & 1.9 & - & - & 1.1 & - & - & - \\
\hline $\begin{array}{l}\text { Cis-threo- } \\
\text { davanafurane }\end{array}$ & 1414 & - & - & - & & - & - & - & - & - & - \\
\hline$\%$ identified & & - & $1.4 \%$ & 1.4 & 1.9 & 0.7 & 0.3 & 1.1 & 2.6 & 0.8 & 2.9 \\
\hline Total identified & & $94.50 \%$ & $92.00 \%$ & $85.30 \%$ & $93.60 \%$ & $88.50 \%$ & $92.70 \%$ & $91.30 \%$ & $89.6 \%$ & $97.00 \%$ & 89.6 \\
\hline
\end{tabular}

${ }^{\mathrm{a}}$ : Percentages obtained by flame ionization detector (FID) peak area normalization (HP-5 column).

${ }^{b}$ : Linear retention indices (DB-5 column).

Table.4 Minimum inhibitory concentrations (MIC) of bacterial growth in the presence of dry arils and peel extracts of five varieties of Punica granatum $\mathrm{L}$. varieties

\begin{tabular}{|c|c|c|c|c|c|c|c|c|c|c|c|}
\hline \multirow[t]{3}{*}{ Strains } & \multicolumn{10}{|c|}{ MIC mg/ml } & \multirow{3}{*}{$\begin{array}{c}\text { MIC's } \\
\text { antibiotics } \\
\text { (mg/ml) } \\
\begin{array}{c}\text { Levofloxaci } \\
\text { n }\end{array}\end{array}$} \\
\hline & \multicolumn{2}{|c|}{ TOUNSI } & \multicolumn{2}{|c|}{ KALAI } & \multicolumn{2}{|c|}{ ZAHRI } & \multicolumn{2}{|c|}{ CHELFI } & \multicolumn{2}{|c|}{ GARSI } & \\
\hline & Peels & Arils & Peels & Arils & Peels & Arils & Peels & Arils & Peels & Arils & \\
\hline \multicolumn{12}{|c|}{ Gram-positive bacteria } \\
\hline $\begin{array}{c}\text { Escherichia } \\
\text { coli ATCC } \\
25922\end{array}$ & 2.5 & 0.62 & 1.25 & 2.5 & 0.62 & 2.5 & 2.5 & 1.25 & 1.25 & 0.62 & 0.61 \\
\hline $\begin{array}{c}\text { P. aeruginosa } \\
\text { ATCC } 27950\end{array}$ & 0.62 & 0.62 & 0.62 & 2.5 & 0.62 & 0.62 & 0.08 & 0.62 & $\begin{array}{c}>10 \mathrm{mg} \\
/ \mathrm{ml}\end{array}$ & 1.25 & 0.4 \\
\hline \multicolumn{12}{|c|}{ Gram-negative bacteria } \\
\hline $\begin{array}{c}\text { Staphylocoсcu } \\
\text { s aureus ATCC } \\
27950\end{array}$ & 0.62 & 0.62 & 1.25 & 2.5 & 0.62 & 1.25 & 0.31 & 1.25 & 1.25 & 2.5 & 0.4 \\
\hline $\begin{array}{c}\text { Enterococcus } \\
\text { faecalis ATCC } \\
29212\end{array}$ & 0.62 & 1.25 & 1.25 & 1.25 & 0.62 & 1.25 & 1.25 & 0.62 & 1.25 & 2.5 & 1.2 \\
\hline
\end{tabular}


Fig.1 Principal components analysis (scores and loading plots, biplot) applied to data set of (a) different phytochemical compounds concentration (b) and antioxidant activity (DPPH, ABTS

$(\%)$ and reducing power (RP: $\mathrm{mg} / \mathrm{ml} \mathrm{AA})$ ), analyzed in different parts of five Tunisian pomegranate varieties (TA: Tounsi Arils, KA: Kalai Arils, ZA: Zahri Arils, CHA: Chelfi Arils, GA: Garsi Arils, TP: Tounsi Peels, KP: Kalai Peels, ZP: Zahri Peels, CHP: Chelfi Peels, GP: Garsi Peels)

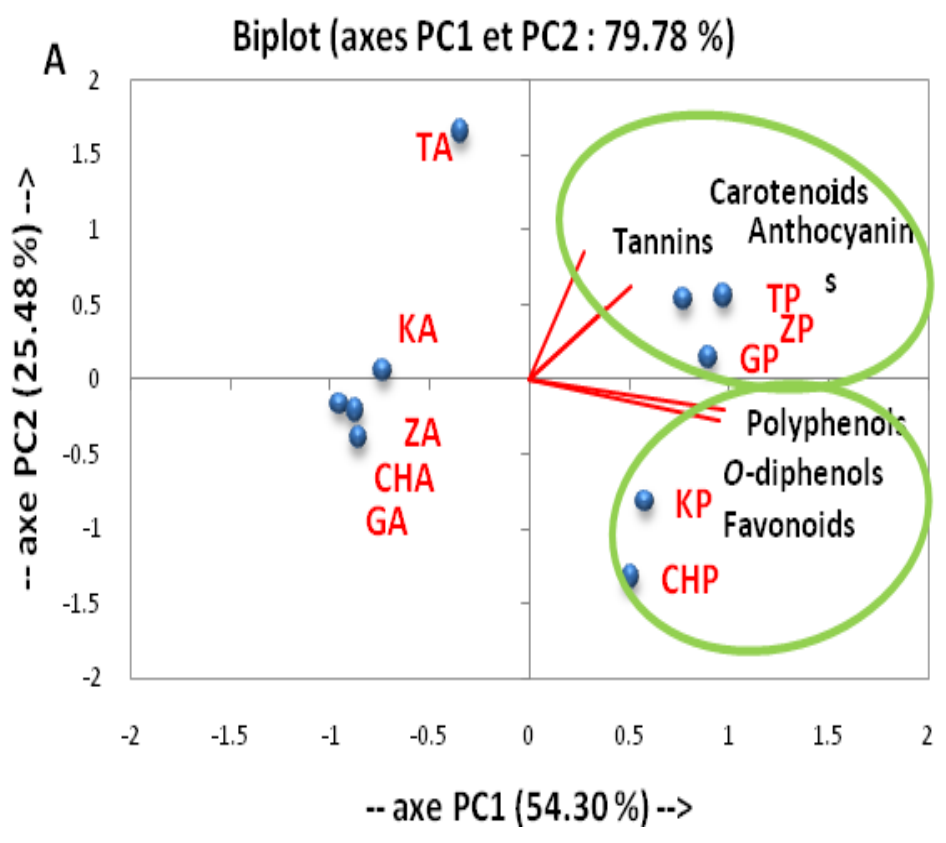

B

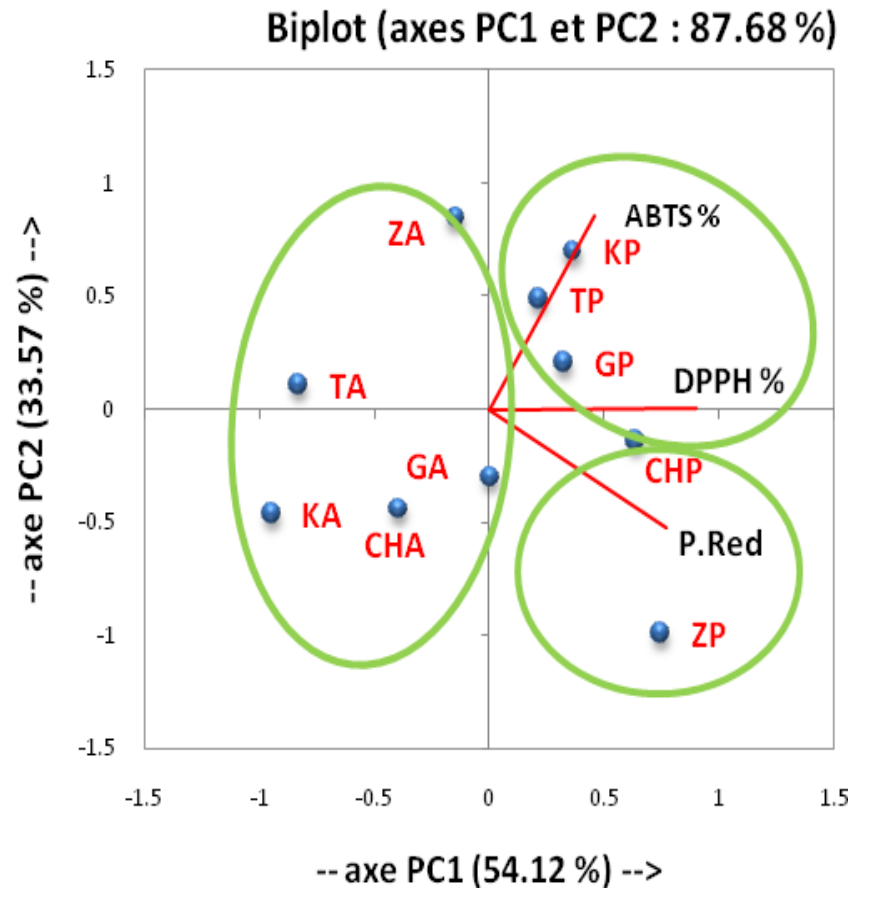


Fig.2 Antioxidant capacity of arils ( $\square$ ) and peel ( $\square$ ) methanolic extracts of five varieties of pomegranate evaluated by (a) DPPH radical scavenging assay, (b) $\mathrm{ABTS}^{+}$cation scavenging assay, and by (c) Reducing Power assay. Values are expressed as means \pm standard deviation (n $=3$ ). Means with different letters were significantly different at the level of $p<0.05$

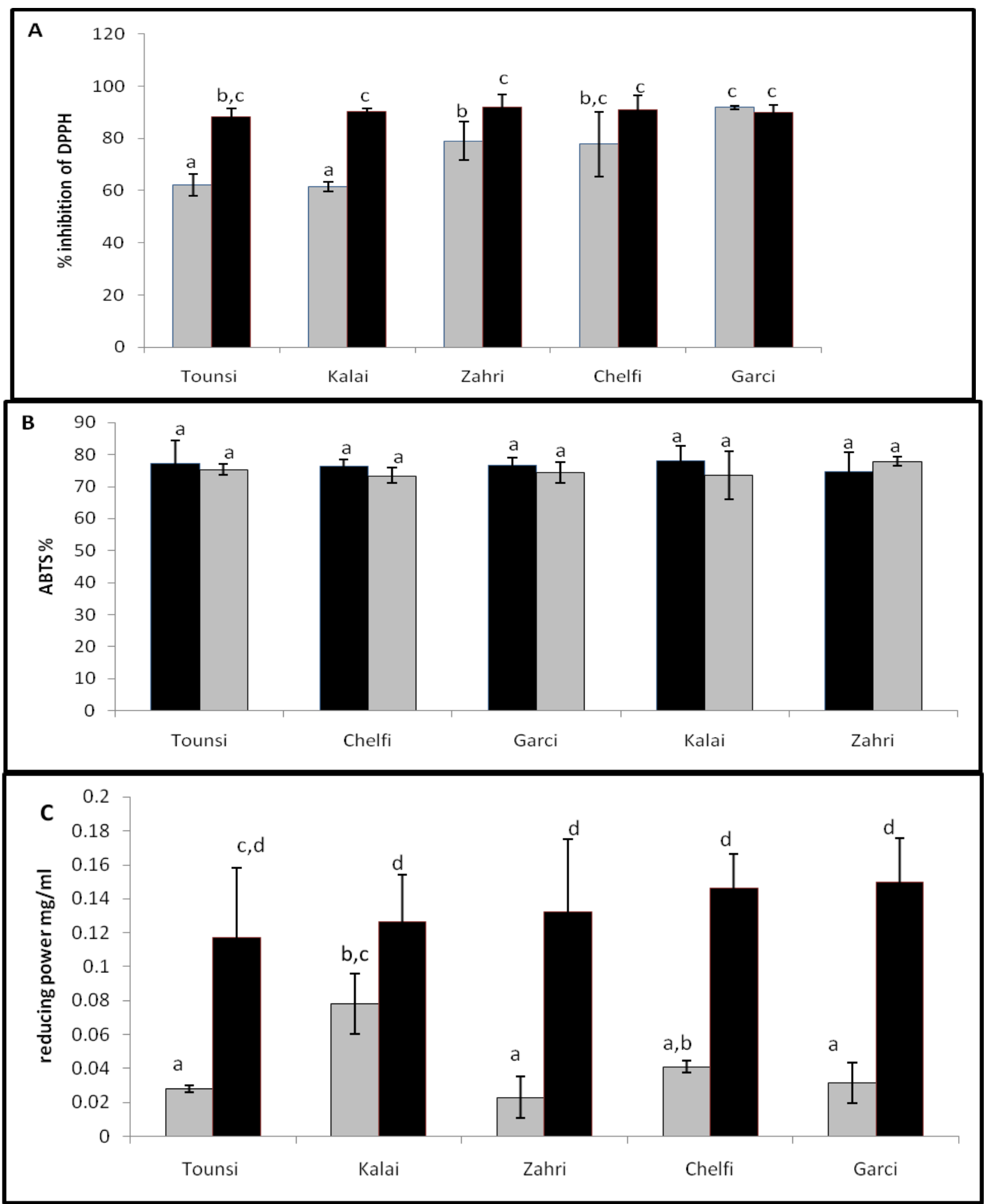


The comparison between varieties showed that peel extracts of Garsi, Chelfi, Zahri and Kalai varieties, have the largest reducing capacity $(0.13$ to $0.15 \mathrm{mg} / \mathrm{ml})$ while Tounsi variety had lowest reducing properties $(0,12$ mg AA / $\mathrm{ml} \mathrm{p} \mathrm{<0,05).} \mathrm{However,} \mathrm{the} \mathrm{arils} \mathrm{are}$ shown to have less effective reducing power. Elfalleh et al., (2011) have reported the reducing capacity of peels, flowers, arils and leaves of some Tunisian varieties of pomegranates. These reducing properties are generally associated with the presence of reductones (Duh, 1998).

Principal Component Analysis (PCA) is conducted to discriminate pomegranate antioxidants by organs (arils / peels) for each studied variety. Three tests are carried out to study the antioxidant power: the power of DPPH, ABTS inhibition and reducing power.

Figure $1 \mathrm{~b}(87.68 \%$ of total variance) reveals that pomegranate peel extracts of all studied varieties are positively correlated with the first axis PC1. In parallel, the three different tests carried out to evaluate the antioxidant capacity of the tested samples, are also positively associated with PC1. In addition, two groups are also separated according to this same axis leading to suggest that peel extracts of Kalai, Tounsi and Garsi varieties have strong antiradical capacity against the ABTS and DPPH radicals. On the other hand, peel extracts of Chelfi and Zahri varieties have an obvious reducing power. However, pomegranate arils extracts are negatively correlated with $\mathrm{PC} 1$, showing the negligible contribution of arils in free radical scavenging and reducing power when compared to pomegranate peels. These latter are endowed with a great antioxidant capacity. These results highlight the importance of valorisation of the inedible parts of the Tunisian pomegranate in terms of antioxidants. Moreover, the antioxidant potential of pomegranate peels could be attributed to their obvious wealth of phenolic compounds that act synergistically to ensure their antioxidant effects. In fact, evident statistic correlations were found between bioactive compounds and antioxidant power including DPPH $(\mathrm{r}=0.5, \mathrm{r}=0.64, \mathrm{r}=0.6, \mathrm{p}<0.01$ for polyphenols, o-diphenols and flavonoids, respectively) and reducing power $(\mathrm{r}=0.64$, $\mathrm{r}=0.9, \quad \mathrm{r}=0.9, \quad \mathrm{p}<0.01$ for polyphenols odiphenols and flavonoids, respectively).Referring to the chemometric analysis (Fig. 2a, 2b); Chelfi and Kalai, varieties (especially in the peel extracts)providing mainly polyphenols, Odiphenols and flavonoids, showed a strong reducing power (for Chelfi) and antiradical capacity against DPPH (Kalai).

\section{Volatile compounds analysis}

A total of 9 classes of volatile chemicals were identified in the volatile extract, including alcohols, esters, aldehydes, terpenoids, ketones, lactones, saturated hydrocarbons, unsaturated hydrocarbons and carboxylic acids. Table 3 shows the chemicals identified in the volatile composition of all the tested pomegranate varieties.

The group of alcohols is formed by a total of 12 compounds which are more abundant in arils (1.6 to $34.7 \%$ ). The 2,3-butandiolis the major compound identified in the volatile extracts $(17.3 \%, 31.5 \%, 1.3 \%$ and $2 \%$, in the arils of Zahri, Chelfi, Garsi and Kalai varieties respectively. This compound is also present in the peels of Zahri and Chelfi (3.8 and $1.3 \%$ ). However, it seems to be totally absent in the variety Tounsi. Phenylethyl alcohol comes second; it is present in arils of four varieties, Zahri (5.3\%), Chelfi (3.2\%), Garsi (1.6\%) and Kalai (1\%). The peels of the Zahri and Chelfi varieties also provide percentages of this alcohol (2.3 and 0.9\%, respectively). Phenylethyl alcohol is the responsible of generation of the honeyed 
odour and probably contributes to the floral aroma of the fruit. In fact aliphatic alcohols are characterized by herbaceous, fruity, citrus and floral scents (Richard, 1992); cis-linalooloxide coming in third place, is present in peels of three varieties, namely Garsi, Zahri and Chelfi (1.5, 1.3 and 1.6\%, respectively). Isopentyl alcohol is found in arils of Tounsi and Zahri varieties (1.6 and $2.4 \%$, respectively).

The esters presented by 31 volatile compounds constitute the major components identified in the volatile fraction. The results provided in Table 3 show that esters are more abundant in arils than peels. Garsi variety has the highest total percentage of esters $(60.9 \%$ in peel level and $54.8 \%$ in arils) while Tounsi variety has the lowest percentage $(27.3 \%$ subdivided between arils and peels). Among all quantified esters, methyl octanoate, methyl decanoate, methyl hexadecanoate, methyl dodecanoate and methyl pentanoateare the most abundant in tested cultivars. In contrast to our findings those methyl esters are much less common in nature than ethyl esters (Tsakiris et al., 2010). Methyl octanoate is present in arils and peels of all studied extracts. Kalai seemed to be the richest cultivar in this ester (44\%) followed by Garsi $(34.8 \%)$ and Zahri (11\%).

Aldehydesform another majority group of volatiles identified in all studied varieties. Their total percentage varied from $20.7 \%$ (Zahri) to $57.9 \%$ (Tounsi). In agreement with our findings Mekni et al., (2013b) confirmed that the Tounsi flowers seemed to be qualitatively the richest in this volatiles group. Quantitatively, aldehydes are more abounded in peels than in arils for all varieties. Among the 19 compounds representing this group, only nanonal volatile was present in all samples. Similarly, the decanal is also found in all tested varieties (except for Chelfiarils). However, the rest of volatiles belonging to this group were distributed with different proportions among the four studied cultivars but totally absent for some ones. These two compounds (decanal, nanonal) are the two linear aldehydes supposed to be responsible for the light sensations of freshness and greenery (Crouzet, 1992).

Among all the studied varieties, Tounsi cultivar seemed to be the richest sample in terpenoids especially in peel (39.7\%). Generally, peels of all cultivars seemed to be the richest part (Table 3). The highest proportion is recorded in Chelfi variety where the total percentage of terpenoids reaches $52.6 \%$, this huge proportion is subdivided between arils $(15.5 \%)$ and peels $(37.1 \%)$ of the same variety. In concordance with our funding Mekni et al., (2013b) demonstrated that the Chelfi flowers seemed to be the richest cultivar in terpenoids $(28.39 \%)$ which confirm that pomegranate fruit conserve some of its volatiles during differentiation from flowers to mature fruit to finally give the pleasant sweet fruity floral taste of pomegranate juice. Interestingly the variety Garsi seemed to be the poorest sample in terpenoids $12.3 \%$ (10.9\% for arils and $1.4 \%$ for peels) which explain his name in Arabic and his sour taste. Volatiles belonging to this group were distributed differently among cultivars and organs (arils and peel). $\gamma$ terpineol presented the major compound of terpenoids $(14.5 \%$ and $5.1 \%$ in Tounsi and Garsi respectively). In addition, trans- $\alpha$ bergamotene, $\beta$-caryophyllene, (E)-eryanyl acetone, limonene, as well as 1,8-cineole, linalool and $\alpha$-terpineol form a minor fraction compared to $\gamma$ - terpineol. Trans- $\alpha$ bergamotene were detected in all the varieties.

Furthermore, we detected the presence of nine ketones, seven saturated hydrocarbons, five unsaturated hydrocarbons, five carboxylic acids where the Tounsi was the richest variety in all these volatile groups. 
Quantitatively, Chelfi is the richest cultivar of some volatile compounds; however, the Tounsi cultivar is characterized by the presence of a multitude of volatile groups and seems to be qualitatively the richest cultivar providing eight groups of volatiles for both peels and arils. However, the rest of cultivars engendered limited types of volatiles. This result clearly indicates that each cultivar can be distinguishable from the other. Aroma compounds accumulate differently depending on the cultivar and that metabolites accumulation depends from the genetically determined sample enzyme composition (Mekni et al., 2013b).

\section{Antibacterial activity}

The antimicrobial proprieties of peel and arils pomegranate extracts of five varieties are assayed against Enterococcus faecalis ATCC 29212, Staphylococcus aureus ATCC 27950, Escherichia coli ATCC 25922 and Pseudomonas aeruginosa ATCC 27950. Table 4 presents minimal inhibitory concentrations (MIC) of bacterial growth in presence of aroma extracts.

Against Escherichia coli ATCC 25922, the Tounsi and Garsiarils extracts and the Zahri peel extracts exhibited highest antibacterial activity among all aroma extracts (MIC $=0.62 \mathrm{mg} / \mathrm{ml}$ ). The most important inhibitory effect recorded for aeruginosa ATCC 27950 is made by the peels extract of Chelfi variety $(0.08 \mathrm{mg} / \mathrm{ml})$ followed by both arils extracts of Chelfi Zahri and Tounsi and peel extracts of Tounsi, Kalai and Zahri $(0.62 \mathrm{mg} / \mathrm{ml})$. While a moderate bactericidal effect is showed for the Garsiarils extract $(1.25 \mathrm{mg} / \mathrm{ml})$ and a low activity is detected in the peel extract for the same variety $(>1.25$ $\mathrm{mg} / \mathrm{ml}$ ).

Interestingly, the pomegranate Chelfi peels extract exhibited a strong inhibitory activity against Staphylococcus aureus ATCC 27950 $(0.31 \mathrm{mg} / \mathrm{ml})$. Also, for the same stain a considerable bactericidal effect is exerts by Tounsi variety and Zahri peels extract $(0.62 \mathrm{mg} / \mathrm{ml})$ and a moderate bactericidal effect showed for the rest of extracts.

Among all used pomegranate extracts, high antibacterial activity was recorded with Tounsi and Zahri peel and Chelfiarils extract $(0.62 \mathrm{mg} / \mathrm{ml})$, whereas a moderate to a low activity is detected in the other extracts (Table 4).

The Gram-positive bacteria were more sensitive to volatiles extract than the gramnegative bacteria, this observation is in concordance with a previous study conducted by Sthéfane et al., (2014) who reported that are the Gram-positive bacteria were more sensitive to ethanol extracts of volatile compounds of Lamiacae. Meanwhile, Mekni et al., (2013b) reported that the volatiles coming from fresh flowers of Tounsi and Chelfi cultivars are more effective against gram negative bacteria than the gram positive. Changes in the organ undergo during its maturation (flower to fruit) and therefore in the composition of the volatile compounds are at the origin of this difference in antibacterial activity, can explain the two different observations.

Moreover, peel and arils pomegranate extracts of Tounsi variety are the most active extracts (Table 4). Indeed, the Tounsi cultivar seemed to be qualitatively the richest, is the cultivar contains the highest number of volatile compounds. Thus, we could suggest that the richness of these volatile compounds is at the origin of this remarkable antibacterial activity. It seems that the relationship between some chemical structures, such as $\alpha$-pinene and linalool and antimicrobial efficacy is present. Indeed, terpenes containing volatile substances are reported to have good 
antimicrobial activity (Dorman and Deans, 2000). Those deductions are in concordance of our finding, indeed in the peel extract of Tounsi variety which is the most active present the highest prevalence of terpenes $(39.7 \%)$ with $1.6 \%$ of linalool. Works focused on the antimicrobial action of the volatile fraction of pomegranate are absent except this conducted by Mekni et al., (2013b) dealing with pomegranate flowers. Brahmi et al., (2011) suggested that the antimicrobial activity of volatile compounds of leaves of $O$. europaea is linked to their content in terpenes.

In the present study, the comparison between peel and arils methanol extracts of five Tunisian pomegranate varieties (Tounsi, Chelfi, Garsi, Zahri, Kalai) showed that peel extracts exhibit highest concentration in phenolics, flavonoids, tannins, anthocyanins and carotenoids beside a high antioxidant power. Among the studied cultivars no one was considered as phytochimical dominant, but we can deduce that the peel extracts of Tounsi, Zahri, and Garsi varieties primarily protect pigmentation compounds such as carotenoids, anthocyanins and tannins. While the Kalai and Chelfi varieties provide mainly polyphenols, O-diphenols and flavonoids. Considerable correlations have been highlighted between polyphenols, Odiphenols and flavonoids of peel extracts and the DPPH scavenging activity as well as reducing power of the same extracts (peels). Those observations motivate us to pay more attention to the non-edible parts (especially peel) and to valorise it for its both therapeutic and nutritional potential.

Volatile compounds accumulated differently according to the cultivar. Quantitatively Chelfi is the richest cultivar of some volatile compounds; however, the Tounsi cultivar seems to be qualitatively the richest cultivar for both peels and arils. The evaluation of the antibacterial activity showed that the Grampositive bacteria were more sensitive to volatiles extract than the gram-negative bacteria. Moreover, pomegranate peel and arils extracts of Tounsi variety are the most active.

Due to the high occurrence of volatiles and phytochemial compounds especially in peels the non-edible part and their considerable antibacterial potential and antioxidant power, those compounds can be used as effective alternatives to treat wide variety of disorders, diseases and malfunctions.

\section{Acknowledgment}

This research was supported by a Grant from the Ministère de l'Enseignement Supérieur et de la Recherche Scientifique LR12ES05 Nutrition-Aliment Fonctionnel et Santé Vasculaire LRNAFS and DGRST-USCR Spectrométrie de masse.

\section{References}

Amri, Z., Zaouay, F., Lazreg-Aref, H., Soltana, H., Mneri, A., Mars, M and Hammami, M. 2017. Phytochemical content, Fatty acids composition and antioxidant potential of different pomegranate parts: Comparison between edible and nonedible varieties grown in Tunisia. Int $\mathbf{J}$ BiolMacromo. 104, 274-280.

Araújo, S. G., Alves L. F., Pinto, M. E. A., Oliveira, G. T., Siqueira, E. P., Ribeiro, R. I. M. A., Ferreira, J. M. S. and Lima, L. A. R. S. 2014. Volatile compounds of Lamiaceae exhibit a synergistic antibacterial activity with streptomycin. Braz J Microbiol. 45, 1341-1347.

Aviram, $\mathrm{M}$ and Rosenblat, M. 2012. Pomegranate protection against cardiovascular diseases. Evidence-Based Complementary Altern Med. doi: $10.1155 / 2012 / 382763$ 
Borochov-Neori, H., Judeinstein, S., Tripler, S., Harari, M., Greenberg, A., Shomer, I and Holland, D. 2009. Seasonal and cultivar variations in antioxidant and sensory quality of pomegranate (Punica granatum L.) fruit. J Food Comp Anal. 22,189-95.

Brahmi, F., Flamini, G., Issaoui, M., Dhibi, M., Dabbou, S., Mastouri, $M$ and Hammami, M. 2011. Chemical composition and biological activities of volatile fractions from three Tunisian cultivars of olive leaves. Med Chem Res. DOI 10.1007/s00044-011-9817-8.

Chung, Y.C., Chen, S.J. Hsu, C.K. Chang, C.T and Chou, S.T. 2005. Studies on the antioxidative activity of Graptopetalum paraguayense E. Walther. Food Chem. 91, 419424

Clevenger, J.F. 1928. Apparatus for the determination of volatile oil. J Am Pharm Assoc. 17: 346-349.

Crouzet, J. 1992. La biogenèse des arômes, in: H. Richard, J. L. Multon (Éds.), Les arômes alimentaires Partie II. Différentes voies de synthèse Coll. Sciences et techniques agro-alimentaires. Paris, France: Lavoisier TEC \& DOC-Apria.

Da Silva, J.A.T., Rana, T.S., Narzary, D., Verma, N., Meshram, D.T and Ranade, S.A.2013. Pomegranate biology and biotechnology: a review, Sci. Hortic. 160: 85-107.

Dorman, H.J.D and Deans, S.G. 2000. Antimicrobial agents from plants: antibacterial activity of plant volatile oils. J Appl. Microbiol. 88, 308-316.

Ferrazzano, G. F., Scioscia, E., Sateriale, D., Pastore, G., Colicchio, R., Pagliuca, C., Cantile, T., Alcidi, B., Coda, M., Ingenito, A., Scaglione, E., Cicatiello, A. G., Volpe. M. G., Di Stasio, M., Salvatore, P and Pagliarulo, C. 2017. In Vitro Antibacterial Activity of Pomegranate Juice and Peel Extracts on Cariogenic Bacteria. Biomed Res Int. 2152749. doi: $10.1155 / 2017 / 2152749$

Gil, M.I., Cherif, J., Ayed, N., Artes, F., TomasBarberan, F. 1995. Influence of cultivar, maturity stage and geographical location on the juice pigmentation of Tunisian pomegranates. Z. Lebensm. Unters. Forsch. 201, 361-364.

Gil, M.I., Tomas-Barberan,F.A., Hess-Pierce, B., Holcroft, D.M. and Kader, A.A. 2000. Antioxidant activity of pomegranate juice and its relationship with phenolic composition and processing. J Agric Food Chem. 48, 4581-4589.

Giusti, M.M and Wrolstad, R.E. 2000. Characterization and measurement of anthocyanins by UV-Visible spectroscopy. In: Giusti MM, Wrolstad RE (eds) Current protocols in Food Analytical Chemistry. Wiley, New York. pp. F1.2.1-F1.2. 13.

Gullon, B., Pintado, M.E. and Viuda-Martos, M. 2016. Assessment of polyphenolic profile and antibacterial activity of pomegranate peel (Punica granatum) flour obtained from coproduct of juice extraction. Food Control. 59, 94-98

Hasnaoui, N., Wathelet, B and Jiménez-Araujo, A. 2014.Valorization of pomegranate peel from 12 cultivars: Dietary fiber composition, antioxidant capacity and functional properties. Food Chem. 160, 196-203.

Khan, I., Rahman, H., Abd El-Salam, N. M., Tawab, A., Hussain A., Ali Khan, T., Ali Khan, U., Qasim, M., Adnan, M., Azizullah, A., Murad, W., Jalal, A., Muhammad, N and Ullah, R. 2017. Punica granatum peel extracts: HPLC fractionation and LC MS analysis to quest compounds having activity against multidrug resistant bacteria. BMC Complementary Altern Med. 17, 247

Kontogiorgis, C and Hadjipavlou-Litina, D. 2005. Synthesis and anti-inflammatory activity of coumarin derivatives. J Med Chem. 48, 6400-8.

Lansky, E. P and Newman, R. A. 2007. Punica granatum (pomegranate) and its potential for prevention and treatment of inflammation and cancer. $J$. Ethnopharmacol.109, 177-206.

Li, X., Wasila, H., Liu, L., Yuan, T., Gao, Z., Zhao, B and Ahmad, I.2015. 
Physicochemical characteristics, polyphenol compositions and antioxidant potential of pomegranate juices from 10 Chinese cultivars and the environmental factors analysis, Food Chem. 175,575584.

Li, Y., Guo, C., Yang, J., Wei, J., Xu, J and Cheng, S. 2006. Evaluation of antioxidant properties of pomegranate peel extract in comparison with pomegranate pulp extract. Food Chem. 96: 254-260.

Li, Y., Guo, C., Yang, J., Wei, J., Xu, J and Cheng, S. 2006. Evaluation of antioxidant properties of pomegranate peel extract in comparison with pomegranate pulp extract. Food Chem. 96,254-260.

Mau, J., Chao, G.R and Wu, K.T. 2001. Antioxidant properties of methanolic extracts from several ear mushrooms. J. Agric. Food. Chem. 49, 5461-5467.

Meerts, I. A. T. M., Verspeek-Rip, C. M., Buskens, C. A. F., Keizer, H. G., Bassaganya-Riera, J., Jouni, Z. E.,, van Huygevoort, A.H., van Otterdijk, F.M and van de Waart, E. J. 2009. Toxicological evaluation of pomegranate seed oil. Food Chem. Toxicol. 47, 1085-1092.

Mekni, M., Azez, R., Tekaya, M., Mechri, B., Hammami, M. 2013a. Phenolic nonphenolic compounds and antioxidant activity of pomegranate flower, leaf and bark extracts of four Tunisian cultivars. J. Med. Plants Res. 7, 1100-1107.

Mekni, M., Flamini, G., Garrab, M., BenHmida, R., Cheraief, I., Mastouri, $\mathrm{M}$ and Hammami, M.2013b. Aroma volatile components, fatty acids and antibacterial activity of four Tunisian Punica granatum L. flower cultivars. Ind Crops Prod. 48, 111- 117

Mekni, M., Dhibi, M., Kharroubi, W., BenHmida, R., Cheraif, and Hammami, M. 2014. Natural conjugated and trans fatty acids in seed oils and phytochemicals in seed extracts issued from three Tunisian pomegranates (Punica granatum. L) cultivars. Int $\mathbf{J}$ CurrMicrobiol App Sci. 3, 778-792.
MiklavčičVišnjevec, A., Ota, A., Skrt, M., Butinar, B., Možina, S.S., Cimerman, N. G., Nečemer, M., Arbeiter, A. B., Hladnik, M., Krapac, M., Ban, D., BučarMiklavčič, M., PoklarUlrih, N. and Bandelj, D. 2017. Genetic, Biochemical, Nutritional and Antimicrobial Characteristics of Pomegranate (Punica granatum L.) Grown in Istria. FoodTechnolBiotechnol. 55, 151-163.

Pagliarulo, C., De Vito, V., Picariello, G., Colicchio, R., Pastore, G., Salvatore, Pand Volpe, M.G. 2016. Inhibitory effect of pomegranate (Punica granatum L.) polyphenol extracts on the bacterial growth and survival of clinical isolates of pathogenic Staphylococcus aureus and Escherichia coli. Food Chem. 190, 824831.

Pagliarulo, C., De Vito, V.,Picariello,G.,Colicchio, R.,, Pastore, G., Salvatore, P and Volpe, M.G.2016. Inhibitory effect of pomegranate (Punica granatum L.) polyphenol extracts on the bacterial growth and survival of clinical isolates of pathogenic Staphylococcus aureus and Escherichia coli. Food Chem., 190, 824-831.

Richard, H. 1992. Connaissance de la nature des arômes. In: Richard, H., Multon, J.L. (Eds.), Les arômes alimentaires, Partie I. Coll. Sciences et techniques agroalimentaires. Tec \& Doc Lavoisier, Paris, pp. 21-37.

Saad, H., Charrier-El Bouhtoury, F., Pizzi, A., Rode, K., Charrier, Band Ayed, N.2012. Characterization of pomegranate peels tannin extractives. Ind Crops Prod. 40, 239-246.

Smania,J.r.A., Smania, E.F.A., DelleMonache, F., Pizzolatti, $\mathrm{M}$ and DelleMonache, G. 2006. Derivatization does not influence antimicrobial and antifungal activi-ties of applanoxidic acids and sterols from Ganoderma spp. Z. Naturforsch. 61,3134.

Stintzing, F. C and Carle,R. 2004. Functional properties of anthocyanins and betalains 
in plants, food and in human nutrition. Trends Food Sci Technol. 15 (1): 19-38

Sun B., Da-Silva J.M. R and Spranger, I. 1998. Critical factors of vanillin assay for catechins and proanthocyanidins. J Agric Food Chem. 46, 4267-4274.

Talcott,S.Tand Howard, L.R.1999. Phenolic autoxidation is responsible for color degradation in processed carrot puree. $\mathbf{J}$ Agric. Food Chem. 47:2109-2115.

Tehranifar, A., Yahya, S., Mahdiyeh, K and Vahid, J.B. 2011. High potential of agroindustrial by-products of pomegranate (Punica granatum L.) as the powerful antifungal and antioxidant substances. Ind. Crop Prod. 34, 1523- 1527

Tehranifar, A., Zarei, M., Nemati, Z., Esfandiyari, B and Vazifeshenas, M.R. 2010.Investigation of physico-chemical properties and antioxidant activity of twenty Iranian pomegranate (Punica granatum L.) cultivars. Sci. Hortic. 126,180-185.

Tomas-Barberan, F.A., Gil, M.I., Cremin, P., Waterhouse, A.L., Hess-Pierce, B and Kader, A.A.2001. HPLC-DAD-ESIMS analysis of phenolic compounds in nectarines, peaches, and plums. J Agric Food Chem. 49, 4748-4760.

Tsakiris, A., Koutinas, A. A., Psarianos, C., Kourkoutas, Y and Bekatorou, A. 2010. A new process for wine production by penetration of yeast in uncrushed frozen grapes. ApplBiochemBiotechnol. 162, 1109-1121.

Tzulker, R., Glazer, I., Bar-Ilan, I., Holland, D., Aviram, $\mathrm{M}$ and Amir, R.2007. Antioxidant activity, polyphenol content, and related compounds in different fruit juices and homogenates prepared from 29 different pomegranate accessions. J Agric Food Chem. 55,9559-70.

Yvonne, V.Y., Dawn, E.B and Meshell, F.C., 2005. Antioxidant activity of dulse (Palmaria palmata) extract evaluated in vitro. Food Chem. 91, 485-494.

Zaouay, F.,Mena, P., Garcia-Viguera, C and Mars, M.2012. Antioxidant activity and physico-chemical properties of Tunisian grown pomegranate (Punica granatum L.) cultivars, Ind Crops Prod. 40, 81-89.

Zhang, L., Fu, Q and Zhang, Y.2011. Composition of anthocyanins in pomegranate flowers and their antioxidant activity. Food Chem. 127, 1444-1449.

Zhishen, J., Mengcheng, T and Jianming, W. 1999. The determination of flavonoid contents in mulberry and their scavenging effects on superoxide radicals. J Agric Food Chem. 64, 555-655.

\section{How to cite this article:}

Manel Mekni, Wafa Kharroubi, Guido Flamimi, Mariem Garrab, Maha Mastouri and Mohamed Hammami. 2018. Comparative Study between Extracts of Different Pomegranate Parts Issued from Five Tunisian Cultivars (Punica granatum L.): Phytochemical Content, Volatile Composition and Biological activity. Int.J.Curr.Microbiol.App.Sci. 7(05): 1663-1682. doi: https://doi.org/10.20546/ijcmas.2018.705.197 